\title{
DETERMINAÇÃO ESPECTROFOTOMÉTRICA POR INJEÇÃo EM FLUXO DE PARACETAMOL (ACETAMINOFENO) EM FORMULAÇÕES FARMACÊUTICAS
}

\author{
Clezio Aniceto e Orlando Fatibello-Filho*
}

Departamento de Química, Centro de Ciências Exatas e de Tecnologia, Universidade Federal de São Carlos, CP 676, 13560-970

São Carlos - SP

Recebido em 4/4/01; aceito em 16/8/01

\begin{abstract}
FLOW INJECTION SPECTROPHOTOMETRIC DETERMINATION OF PARACETAMOL (ACETAMINOPHEN) IN PHARMACEUTICAL FORMULATIONS. A flow injection spectrophotometric procedure is proposed for the determination of paracetamol (acetaminophen) in pharmaceutical formulations. Powdered and liquid samples were previously dissolved/diluted in $0.05 \mathrm{~mol} \mathrm{~L}^{-1}$ hydrochloric acid solution and a volume of $250 \mu \mathrm{L}$ was injected directly into a carrier stream of this same acid solution, flowing at $2.5 \mathrm{~mL} \mathrm{~min}^{-1}$. Paracetamol reacts with sodium hypochlorite forming $\mathrm{N}$-acetyl-p-benzoquinoneimine which then reacts with sodium salicylate in sodium hydroxide solution yielding a blue indophenol dye which was measured at $640 \mathrm{~nm}$ in the $\mathrm{pH}$ range of 9.5-10.0. Paracetamol was determined in pharmaceutical products in the 1.0 to $100.0 \mathrm{mg} \mathrm{L}^{-1}\left(3.3 \times 10^{-6}\right.$ a $\left.6.6 \times 10^{-4} \mathrm{~mol} \mathrm{~L}^{-1}\right)$ concentration range, with a detection limit of $0.5 \mathrm{mg} \mathrm{L}^{-1}\left(1.6 \times 10^{-6} \mathrm{~mol} \mathrm{~L}^{-1}\right)$. The recovery of this analyte in five samples ranged from 98.0 to $103.6 \%$. The analytical frequency was 80 determinations per hour and the RSDs were less than $1 \%$ for paracetamol concentrations of $25.0,50.0$ and $75.0 \mathrm{mg} \mathrm{L}^{-1}(\mathrm{n}=10)$. A paired t-test showed that all results obtained for paracetamol in commercial formulations using the proposed flow injection procedure and a spectrophotometric batch procedure agree at the $95 \%$ confidence level.
\end{abstract}

Keywords: paracetamol; flow injection spectrophotometry; pharmaceutical formulations.

\section{INTRODUÇÃO}

Paracetamol é um analgésico-antipirético pertencente à classe dos derivados do p-aminofenol, introduzido no século passado como resultado de pesquisas destinadas a substitutos para acetanilida. Embora possuam propriedades analgésico-antipiréticas, a fenacetina e a acetanilida dão origem à metemoglobina (hemoglobina incapaz de transportar oxigênio), devido à formação de um precursor da anilina. Tanto a acetanilida quanto a fenacetina são metabolizadas a paracetamol, a substância ativa. Outros fármacos dessa classe são a anidoxina, butacetina, etoxazena, fenacetinol, parapropanol e parsalmida ${ }^{1}$.

O paracetamol é obtido por acetilação do p-aminofenol com ácido acético glacial e anidrido acético. Esse fármaco se apresenta como um pó branco, inodoro e ligeiramente hidrossolúvel e as suas atividades analgésicas e antipiréticas são similares às da acetanilida e fenacetina, da qual é metabólito. O paracetamol não tem atividade anti-inflamatória, mas ainda assim é provavelmente o antipiréticoanalgésico de segunda escolha, sobretudo para pacientes alérgicos ao ácido acetilsalicílico ou que sofram de úlceras pépticas. Por ser menos tóxico que a fenacetina, o paracetamol acabou substituindo-a em diversas formulações farmacêuticas, sendo habitual administrar doses diárias ${ }^{1}$ variando de 0,3 a $1 \mathrm{~g}$.

A Farmacopéia Americana (USP-23) 2 recomenda a cromatografia em camada delgada e espectrofotometria para a determinação de paracetamol em preparações farmacêuticas e a A.O.A.C ${ }^{3}$ o método espectrofotométrico com detecção em $520 \mathrm{~nm}$.

Há citados na literatura diversos métodos para a determinação de paracetamol, dentre estes destacam-se os métodos microbiológico ${ }^{4}$,

*e-mail: bello@dq.ufscar.br volumétricos ${ }^{5}$, por quimioluminescência ${ }^{6}$, espectrofotométrico ${ }^{7}$, fluorimétrico ${ }^{8}$, eletroanalíticos ${ }^{9-13}$, espectroeletroquímico ${ }^{14}$, cromatográficos ${ }^{15-19}$ e em análise por injeção em fluxo por espectrofotometria no $\mathrm{UV}^{20}$ e visível ${ }^{21-23}$, fluorescência ${ }^{24}$, quimioluminescência ${ }^{25}$, biamperometria ${ }^{26,27}$ e espectrofotometria FTIR (do inglês: Fourier transform infrared $)^{28}$.

A análise, o conhecimento e a distribuição de analgésicoantipiréticos em produtos farmacêuticos tem-se constituído um dos principais problemas desde que se iniciou a investigação destes compostos. O maior interesse nestas análises está centrado nos produtos naturais, na necessidade destes compostos em seres humanos e seu emprego na indústria farmacêutica.

O desenvolvimento de novos procedimentos para determinação dessa classe de fármacos, especialmente aqueles que apresentam em suas formulações antiinflamatórios baseados em substâncias que atuam como imunoterápicos (imunossupressores ou imunopotenciadores), se deve ao fato de seu crescente uso na indústria farmacêutica entre outras, que necessitam de procedimentos rápidos, de baixo custo, precisos e alta seletividade para a determinação destes fármacos, seja na forma livre ou na presença de outras substâncias, devido ao crescimento exagerado da demanda destes produtos ocasionado pelo crescimento populacional em países em desenvolvimento e o crescimento das doenças relacionadas a terceira idade.

Desenvolveu-se neste trabalho um procedimento em fluxo com detecção espectrofotométrica para a determinação de paracetamol (acetaminofeno) em preparações farmacêuticas. Nesse procedimento, o paracetamol é oxidado com hipoclorito de sódio formando o Nacetil-p-benzoquinonaimina que reage com salicilato de sódio em solução básica produzindo um composto indofenólico ${ }^{29}(\mathrm{~N}-[\mathrm{p}-$ hidroxifenil]-p-benzoquinonaimina) com forte absorção molecular em $640 \mathrm{~nm}$, no intervalo de pH entre 9,5 e 10,0, conforme esquema mostrado na Figura 1. 


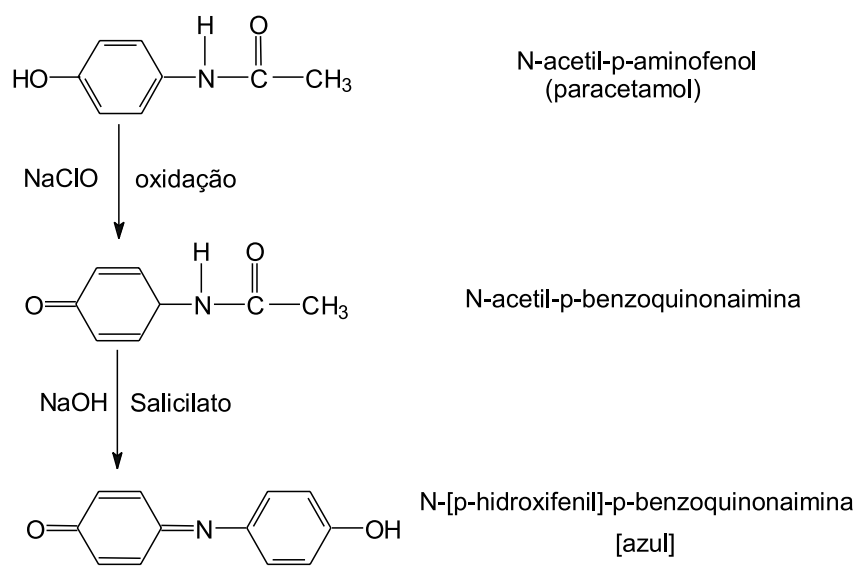

Figura 1. Esquema da reação de oxidação do paracetamol (acetaminofeno) com hipoclorito de sódio 0,30\% (v/v), salicilato de sódio $8,0 \%(\mathrm{~m} / \mathrm{v}) \mathrm{em}$ hidróxido de sódio $0,5 \mathrm{~mol} L^{-1}$ produzindo um composto indofenólico de coloração azul $2^{29}$ com forte absorção em $640 \mathrm{~nm}$

\section{PARTE EXPERIMENTAL}

\section{Equipamentos}

Todas as medidas espectrofotométricas foram feitas em um espectrofotômetro Femto, modelo 435 com um célula de quartzo (caminho óptico $1,0 \mathrm{~cm}$ ) conectado a um registrador Cole Parmer (Niles, IL, USA, modelo 12020000 de dois canais). O efeito da temperatura na reação de oxidação do paracetamol foi feito usando-se um banho termostático Tecnal, modelo TE 184. Para propulsão das soluções de referência, reagentes e amostras utilizou-se uma bomba peristáltica Ismatec (Zurich, Switzerland, modelo 7618-40) e tubos de bombeamento de Tygon de diversos diâmetros internos. As soluções das amostras e de referência foram introduzidas no sistema FIA, utilizando-se um comutador automático Micronal, modelo B352 e conectores de acrílico foram utilizados na montagem de todo o sistema de injeção em fluxo (Figura 2).

Para dissolução e solubilização das amostras utilizou-se um sonificador (Ultra som, Unique, modelo USC 1400).

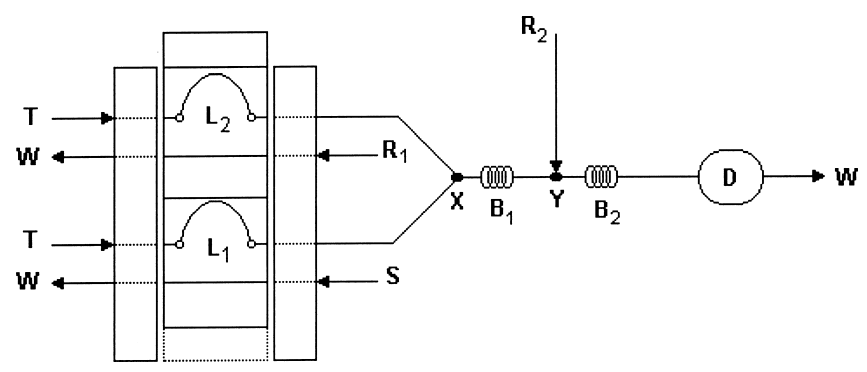

Figura 2. Diagrama de fluxos do sistema para determinação espectrofotométrica de paracetamol. As três peças retangulares representam um esquema do injetor-comutador; $\boldsymbol{S}$, soluções de referência ou de amostra; $\boldsymbol{L}_{\boldsymbol{l}}$, alça de amostragem $(50 \mathrm{~cm}, 250 \mu \mathrm{L}) ; \boldsymbol{L}_{2}$, alça $(12,5 \mathrm{~cm} ; 62,5 \mu \mathrm{L}), \boldsymbol{R}_{\boldsymbol{I}}$ reagente ( $\mathrm{NaClO} 0,30 \%(\mathrm{v} / \mathrm{v})$ ); $\boldsymbol{R}_{2}$ reagente (salicilato de sódio 8,0\% $(\mathrm{m} / \mathrm{v}$ )

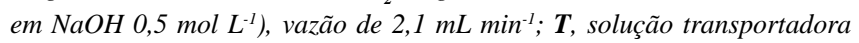
( $\mathrm{HCl}$ 0,05 $\mathrm{mol} \mathrm{L}^{-1}$ ) com vazão de $2,5 \mathrm{~mL} \mathrm{~min}^{-1}$; D, espectrofotômetro em 640 $n m ; \boldsymbol{X} \boldsymbol{e} \boldsymbol{Y}$ confluências; $\boldsymbol{B}_{1}$ reator helicoidal $\left(100 \mathrm{~cm}, 0,8 \mathrm{~mm}\right.$ d.i.); $\boldsymbol{B}_{2}$ reator helicoidal (200 cm, 0,8 $\mathrm{mm}$ d.i.); $\boldsymbol{W}$, descarte
As medidas espectrofotométricas (método comparativo) foram feitas com um espectrofotômetro Hewlett Packard (USA, modelo 8452 A).

\section{Reagentes e soluções}

Paracetamol (acetaminofeno), reagente de grau bioquímico, foi utilizado para preparação das soluções de referência. Os demais reagentes utilizados foram de grau analítico e a água empregada na preparação das soluções foi proveniente de um sistema Milli-Q Millipore (Redford, MA, USA, modelo UV Plus Ultra-baixo teor de compostos orgânicos dissolvidos). Sacarose, glicose, lactose, ácido cítrico, ácido ascórbico, amido, sorbitol, sacarina, ciclamato, cafeina, polietileno glicol e etileno glicol foram adquiridos da Sigma Chemical Co (St. Louis, MO, USA). A solução estoque de paracetamol 200 $\mathrm{mg} \mathrm{L}^{-1}$ foi preparada em $\mathrm{HCl} 0,05 \mathrm{~mol} \mathrm{~L}^{-1}$, sendo então as soluções de referência desse fármaco nas concentrações de 1,0 a $100,0 \mathrm{mg} \mathrm{L}^{-1}$ preparadas diariamente em $\mathrm{HCl} 0,05 \mathrm{~mol} \mathrm{~L}^{-1}$, por diluições apropriadas da solução estoque. Essas soluções foram protegidas da luz, sendo acondicionadas em frascos de cor âmbar. Prepararam-se diariamente soluções de hipoclorito de sódio $0,30 \%$ (v/v) e salicilato de sódio $8,0 \%(\mathrm{~m} / \mathrm{v})$ em hidróxido de sódio $0,5 \mathrm{~mol} \mathrm{~L} \mathrm{~L}^{-1}$.

\section{Preparação das amostras}

Paracetamol foi determinado em amostras sólidas (pó), gotas e elixir, adquiridos em farmácias locais, comercializadas como Descon (Merrell Lepetit Farmacêutica e Industrial Ltda, Santo Amaro, SP), Resprin (Johnson \& Johnson Indústria e Comércio Ltda, São José dos Campos, SP), Resfenol (Laboratórios Galenogal Ltda, Porto Alegre, RS), Tylenol (Cilag Farmacêutica Ltda, São José dos Campos, SP) e Vick Pyrena (Procter \& Gamble do Brasil S.A., Louveira, SP).

\section{a) amostras sólidas}

Uma massa acuradamente pesada entre 15 a $30 \mathrm{mg}$ de cada amostra sólida (pó) foi transferida para um béquer de $250 \mathrm{~mL}$ contendo 200 $\mathrm{mL}$ de solução de $\mathrm{HCl} 0,05 \mathrm{~mol} \mathrm{~L}^{-1}$ e então sonificada por $30 \mathrm{~min}$. Após esse tempo, cada uma das suspensões foi filtrada em papel de filtro Whatman № 1 e o filtrado transferido para um balão de $250 \mathrm{~mL}$, sendo em seguida o volume completado com solução $\mathrm{HCl}$ 0,05 mol L-1.

\section{b) amostras líquidas}

Aliquotas entre 12,5 a $63,0 \mu \mathrm{L}$ das amostras líquidas foram transferidas para balões de $50 \mathrm{~mL}$ e os volumes completados com solução de $\mathrm{HCl} 0,05 \mathrm{~mol} \mathrm{~L}^{-1}$

\section{Procedimento espectrofotométrico}

O procedimento espectrofotométrico proposto pela Farmacopéia Brasileira $^{30}$ foi utilizado para a determinação dos teores de paracetamol nas cinco amostras comerciais.

\section{Sistema de análise por injeção em fluxo}

A Figura 2 apresenta o esquema do sistema de análises por injeção em fluxo utilizado com detecção espectrofotométrica na posição de análise. Nesse procedimento as soluções de referência ou de amostra (S) do paracetamol foram introduzidas no fluxo transportador $(\mathbf{T}$, $\mathrm{HCl}$ 0,05 mol L ${ }^{-1}$, vazão de $2,5 \mathrm{~mL} \mathrm{~min}^{-1}$ ) conjuntamente com $\mathrm{NaClO}$ $0,30 \%(\mathrm{v} / \mathrm{v})\left(\mathbf{R}_{\mathbf{1}}\right)$, através de alças de $50 \mathrm{~cm}\left(\mathbf{L}_{\mathbf{1}}, 250 \mu \mathrm{L}\right)$ e de 12,5 $\mathrm{cm}\left(\mathbf{L}_{2}, 62,5 \mu \mathrm{L}\right)$, respectivamente, confluindo em $\mathrm{X}$ (ponto de con- 
fluência). No reator $\mathbf{B}_{1}$, há a formação de N-acetil-p-benzoquinonaimina, que na sequência recebe, no ponto de confluência $\mathbf{Y}$, solução de salicilato de sódio $8,0 \%(\mathrm{~m} / \mathrm{v})$ em hidróxido de sódio 0,5 mol L-1 $\left(\mathbf{R}_{2}\right)$, a uma vazão de $2,1 \mathrm{~mL} \mathrm{m^{-1 }}$, formando então no reator $\mathbf{B}_{2}$ a N-[p-hidroxifenil]-p-benzoquinonaimina, um composto indofenólico estável de coloração azul intensa, que foi monitorado espectrofotometricamente em $640 \mathrm{~nm}$ (D).

\section{RESULTADOS E DISCUSSÃO}

\section{Efeito da concentração dos reagentes}

Estudou-se o efeito das concentrações de ácido clorídrico no intervalo de concentrações de 0,01 a $0,3 \mathrm{~mol} \mathrm{~L}^{-1}$, do salicilato de sódio de 2,0 a 10,0\% (m/v) em hidróxido de sódio de 0,1 a $1,0 \mathrm{~mol} \mathrm{~L}^{-1}$ e do hipoclorito de sódio de 0,05 a $0,5 \%$ (v/v) sobre o sinal analítico. O melhor sinal analítico foi obtido em ácido clorídrico $0,05 \mathrm{~mol} \mathrm{~L}^{-1}$, salicilato de sódio $8,0 \%(\mathrm{~m} / \mathrm{v})$ em hidróxido de sódio $0,5 \mathrm{~mol} \mathrm{~L} \mathrm{~L}^{-1} \mathrm{e}$ hipoclorito de sódio $0,30 \%(\mathrm{v} / \mathrm{v})$.

\section{Efeito do volume das alças de amostragem}

Estudou-se inicialmente o efeito do volume da alça de amostragem $\mathbf{L}_{1}$ de 125 a $750 \mu \mathrm{L}(25$ a $150 \mathrm{~cm})$ para soluções de referência de paracetamol de $25,50,75$ e $100 \mathrm{mg} \mathrm{L}^{-1}$, fixando-se a concentração de ácido clorídrico em $0,05 \mathrm{~mol} \mathrm{~L}^{-1}$ (solução transportadora), do salicilato de sódio 8,0\% (m/v) em hidróxido de sódio 0,5 mol $\mathrm{L}^{-1}$, de hipoclorito de sódio $0,30 \%$ (v/v), vazão constante de 2,5 $\mathrm{mL} \mathrm{min}^{-1}$ e alça $\mathbf{L}_{2}$ para o reagente hipoclorito de sódio $0,30 \%(\mathrm{v} / \mathrm{v})$ em $125 \mu \mathrm{L}$ sobre o sinal analítico. Observou-se para a alça $\mathbf{L}_{1}$, que o sinal analítico aumentou até volume de $500 \mu \mathrm{L}$, mantendo-se praticamente constante em volumes superiores. No estudo do efeito do volume da alça $\mathbf{L}_{2}$, variando de 62,5 a $500 \mu \mathrm{L}(12,5$ a $100 \mathrm{~cm})$, utilizaram-se as mesmas condições experimentais mencionadas e a alça $\mathbf{L}_{\mathbf{1}}$ de $500 \mu \mathrm{L}$. Nessas condições, o sinal analítico aumentou de magnitude até volume de $125 \mu \mathrm{L}$, decrescendo suavemente para volumes da alça $\mathbf{L}_{2}$ superiores. Assim utilizaram-se alças de $500 \mu \mathrm{L}\left(\mathbf{L}_{\mathbf{1}}\right)$ e $125 \mu \mathrm{L}\left(\mathbf{L}_{2}\right)$ para o desenvolvimento desse trabalho.

Trabalhando-se com a razão volumétrica de 1:4 das alças $\mathbf{L}_{2}$ e $\mathbf{L}_{1}$, encontrou-se a mesma sensibilidade do procedimento em fluxo. Objetivando economia de reagentes e diminuição do volume de descarte do sistema em fluxo, optou-se em trabalhar com alças de $50 \mathrm{~cm}$ $(250 \mu \mathrm{L})$ para as soluções de referência e amostras e de $12,5 \mathrm{~cm}$ $(62,5 \mu \mathrm{L})$ para a alça do reagente $\mathbf{R}_{1}$, hipoclorito de sódio $0,30 \%(\mathrm{v} /$ $\mathrm{v})$, sem causar perda significativa da sensibilidade no sinal analítico.

\section{Efeito das vazões}

Estudou-se o efeito da vazão de 1,$7 ; 2,1 ; 2,5 ; 3,1 ; 3,6$ e 4,4 mL $\min ^{-1}$ para a solução transportadora $\mathbf{T}, \mathrm{HCl} 0,05 \mathrm{~mol} \mathrm{~L}^{-1}$, nas condições descritas na Figura 2. Observou-se um aumento significativo da magnitude do sinal analítico até a vazão de $2,5 \mathrm{~mL} \mathrm{~min}^{-1}$ da solução transportadora, decrescendo para vazões superiores, devido provavelmente ao aumento da dispersão da zona de amostra. Sendo assim, empregou-se essa vazão nos estudos seguintes.

Estudou-se também o efeito da vazão de 0,$4 ; 0,7 ; 0,9 ; 1,1 ; 1,7$ e 2,5 mL min $\mathrm{ma}^{-1}$ da solução de salicilato de sódio $8,0 \%(\mathrm{~m} / \mathrm{v}) \mathrm{em} \mathrm{NaOH}$ $0,5 \mathrm{~mol} \mathrm{~L}^{-1}$ sobre o sinal analítico, mantendo-se uma vazão de 2,5 $\mathrm{mL} \mathrm{min}^{-1}$ da solução transportadora de $\mathrm{HCl} 0,05 \mathrm{~mol} \mathrm{~L}^{-1} \mathrm{e}$ demais condições descritas na legenda da Figura 2. Observou-se um aumento significativo do sinal analítico até a vazão de $2,1 \mathrm{~mL} \mathrm{~min}{ }^{-1}$ da solução transportadora, decrescendo para vazões superiores. Esse efeito pode estar relacionado com a diluição da zona de amostra e também com a velocidade de reação do salicilato e N-acetil-pbenzoquinonaimina. O desenvolvimento da reação, a partir da vazão 2,1 $\mathrm{mL} \mathrm{min}^{-1}$ da solução de salicilato de sódio, não compensou a perda devida a diluição desses reagentes. Desta maneira, utilizou-se uma vazão de 2,5 $\mathrm{mL} \mathrm{min}^{-1}$ para a solução transportadora da amostra ou soluções de referência e vazão de $2,1 \mathrm{~mL} \mathrm{~min}^{-1}$ para a solução de salicilato de sódio $8,0 \%(\mathrm{~m} / \mathrm{v})$ em hidróxido de sódio $0,5 \mathrm{~mol} \mathrm{\textrm {L } ^ { - 1 }}$.

\section{Efeito dos comprimentos das bobinas reacionais}

Estudou-se o efeito do comprimento da bobina de reação $\mathbf{B}_{1}$ de $50,75,100$ e $150 \mathrm{~cm}$, sobre o sinal analítico. Observou-se que para os comprimentos de 100 e $150 \mathrm{~cm}$ os sinais obtidos foram praticamente constantes, não havendo perda significativa de sinal analítico. Para os comprimentos de 50 e $75 \mathrm{~cm}$ foi observado uma perda considerável do sinal, devido possivelmente a não completa homogeinização dos reagentes injetados em confluência e/ou velocidade da reação que não estaria compensando a perda da diluição da zona de amostra. Portanto, utilizou-se a bobina de $100 \mathrm{~cm}$ para a conclusão do trabalho.

Investigou-se também o efeito do comprimento da bobina de reação $\mathbf{B}_{2}$ de $50,75,100,150,200,250$ e $300 \mathrm{~cm}$ sobre o sinal analítico. Observou-se que para os comprimentos de 200, 250 e 300 $\mathrm{cm}$ os sinais obtidos foram praticamente constantes não havendo perda significativa de sinal analítico, tendo assim atingido a máxima concentração do azo composto formado nas condições experimentais do trabalho. Para os comprimentos de 50, 75, 100 e $150 \mathrm{~cm}$ foi observado uma perda considerável do sinal, devido provavelmente ao baixo rendimento da reação global de formação do azo composto, em virtude do menor tempo de residência do salicilato com o Nacetil-p-benzoquinonaimina formado na oxidação do paracetamol com hipoclorito de sódio. Portanto, utilizou-se a bobina de $200 \mathrm{~cm}$ para a conclusão do trabalho.

\section{Curva analítica}

A curva analítica foi obtida por injeções sucessivas em triplicata de volumes de soluções de referência de paracetamol (acetaminofeno) na faixa de concentrações de 1,0 a $100,0 \mathrm{mg} \mathrm{L}^{-1}$, preparadas diariamente em $\mathrm{HCl} 0,05 \mathrm{~mol} \mathrm{~L}^{-1}$, vazões de $2,5 \mathrm{~mL} \mathrm{~min}^{-1}$ e 2,1 $\mathrm{mL} \mathrm{min}^{-1}$ para a solução transportadora $\mathbf{T}\left(\mathrm{HCl} 0,05 \mathrm{~mol} \mathrm{~L}^{-1}\right.$ e solução de salicilato de sódio $8,0 \%(\mathrm{~m} / \mathrm{v})$ em hidróxido de sódio $0,5 \mathrm{~mol} \mathrm{~L} \mathrm{~L}^{-1}$, respectivamente, alças de amostragem $\left(\mathbf{L}_{\mathbf{1}}\right)$ para amostras e soluções de referência de $50 \mathrm{~cm}(250 \mu \mathrm{L})$ e alça $\left(\mathbf{L}_{2}\right)$ para o reagente $\mathrm{NaClO}$ $0,30 \%(\mathrm{v} / \mathrm{v})$ de $12,5 \mathrm{~cm}(62,5 \mu \mathrm{L})$ e bobinas reacionais $\mathbf{B}_{1}$ de $100 \mathrm{~cm}$ e $\mathbf{B}_{2}$ de $200 \mathrm{~cm}$. A curva analítica obtida pode ser descrita pela equação: $\mathbf{A}=(-5,85 \pm 0,09) \times 10^{-3}+(6,06 \pm 0,06) \times 10^{-3} \mathbf{C} ; r=0,9998$, onde A é a absorbância e $\mathbf{C}$ a concentração do paracetamol em $\mathrm{mg} \mathrm{L}^{-1}$. A curva analítica foi linear na faixa de concentração de paracetamol de 1,0 a $100 \mathrm{mg} \mathrm{L}^{-1}\left(3,3 \times 10^{-6}\right.$ a $\left.6,6 \times 10^{-4} \mathrm{~mol} \mathrm{~L}^{-1}\right)$ e limite de detecção de $0,5 \mathrm{mg} \mathrm{L}^{-1}\left(1,6 \times 10^{-6} \mathrm{~mol} \mathrm{~L}^{-1}\right)$ (três vezes o desvio padrão do branco/ inclinação da curva analítica). A frequência analítica foi de $80 \mathrm{~h}^{-1} \mathrm{e}$ os sinais transientes obtidos para as soluções de referência de paracetamol e soluções de amostras são apresentados na Figura 3.

O sistema FIA proposto apresentou maiores linearidade e sensibilidade que aqueles sistemas descritos na literatura com detecção espectrofotométrica no $\mathrm{UV}^{20}$ e no visível ${ }^{21-23}$, fluorescência ${ }^{24}$, quimioluminescência ${ }^{25}$, biamperométrica ${ }^{26,27}$ e FTIR $^{28}$, além de maior freqüência analítica ${ }^{20,21,23-25,26}$.

\section{Estudo da repetibilidade}

Estudou-se a repetibilidade do procedimento proposto para concentrações de 25,0 e 50,0 $\mathrm{mg} \mathrm{L}^{-1}$ de paracetamol, obtendo-se desvios 


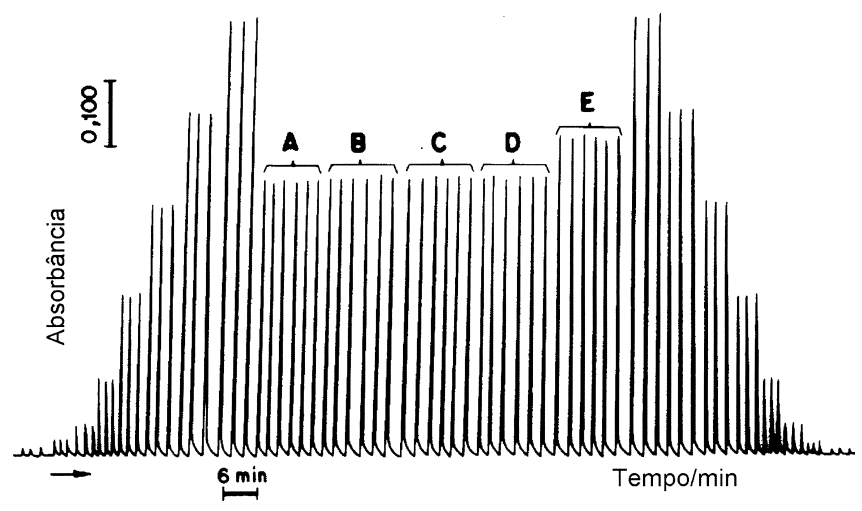

Figura 3. Sinais transientes obtidos em triplicata para soluções de referência de paracetamol (acetaminofeno) nas concentrações de 1,0; 5,0; 10,0; 25,0; 35,0; 50,0; 75,0 e 100,0 $\mathrm{mg} \mathrm{L}^{-1}$ e em sextuplicata para soluções de amostras (A, Descon; B, Resfenol; C, Resprin; D, Tylenol; E, Vick Pyrena) e soluções de referência novamente. As condições do sistema de injeção em fluxo são as mesmas especificadas na legenda da Figura 2

padrão relativos menores que $2 \%$ para as concentrações estudadas $(n=10)$.

\section{Estudo da seletividade e de adição/recuperação de paracetamol em produtos farmacêuticos}

O efeito de algumas substâncias geralmente presentes nas amostras comerciais como sacarose, glicose, lactose, ácido cítrico, amido, sorbitol, sacarina, ciclamato, cafeina, polietileno e etileno glicol foram estudados como interferentes em potencial. Nenhum desses compostos investigados nas razões de concentrações de 10:1; 1:1 e 1:10 causou interferência no procedimento em fluxo.

A Tabela 1 apresenta os resultados da adição e recuperação do paracetamol (acetaminofeno) em fármacos para três concentrações:

Tabela 1. Estudo de adição e recuperação de paracetamol em amostras de preparações farmacêuticas para três diferentes concentrações

\begin{tabular}{lccc}
\hline \multirow{2}{*}{ Amostra } & \multicolumn{2}{c}{ Paracetamol $\left(\mathrm{mg} \mathrm{L}^{-1}\right)$} & \\
& Adicionado & Recuperado* & Recuperação (\%) \\
\hline Descon & 25,0 & $24,9 \pm 0,5$ & 99,6 \\
& 50,0 & $50,0 \pm 0,4$ & 100,0 \\
& 75,0 & $75,4 \pm 0,7$ & 100,5 \\
Resfenol & 25,0 & $25,8 \pm 0,8$ & 103,2 \\
& 50,0 & $50,7 \pm 0,6$ & 101,4 \\
& 75,0 & $75,9 \pm 0,6$ & 101,2 \\
Resprin & 25,0 & $25,9 \pm 0,8$ & 103,6 \\
& 50,0 & $50,9 \pm 0,7$ & 101,8 \\
& 75,0 & $76,1 \pm 0,9$ & 101,5 \\
Tylenol & 25,0 & $25,6 \pm 0,6$ & 102,4 \\
& 50,0 & $51,5 \pm 0,8$ & 103,0 \\
& 75,0 & $76,1 \pm 0,8$ & 101,5 \\
& & & \\
Vick Pyrena & 25,0 & $24,5 \pm 0,6$ & 98,0 \\
& 50,0 & $50,5 \pm 0,8$ & 101,0 \\
& 75,0 & $75,7 \pm 0,6$ & 100,9 \\
\hline
\end{tabular}

* média \pm estimativa do desvio padrão de 6 medidas
25,0; 50,0 e 75,0 $\mathrm{mg} \mathrm{L}^{-1}$ de soluções de amostras de paracetamol, obtendo-se fatores de recuperações entre $98,0 \%$ e $103,6 \%$. Concluise dos resultados obtidos que não houve interferência da matriz desses produtos comerciais no método analítico proposto.

\section{Aplicação}

A Tabela 2 apresenta uma comparação entre os teores de paracetamol encontrados nessas preparações farmacêuticas utilizando-se um método oficial ${ }^{30}$ e o procedimento por injeção em fluxo proposto. Aplicando-se o teste t-pareado para os resultados obtidos utilizando-se os dois procedimentos, obteve-se t igual a 2,24; valor menor que aquele tabelado $\left(\mathrm{t}_{0,05 / 2,4}=2,78\right)$, indicando assim que não há diferença significativa entre os resultados a um nível de $95 \%$ de confiança.

Tabela 2. Determinação de paracetamol em preparações farmacêuticas usando o método espectrofotométrico e espectrofotométrico por injeção em fluxo proposto

\begin{tabular}{|c|c|c|c|}
\hline \multirow[t]{2}{*}{ Amostras } & \multicolumn{2}{|c|}{$\begin{array}{c}\text { Paracetamol }(\mathrm{mg} / \mathrm{g})^{*} \\
(\mathrm{mg} / \mathrm{mL})\end{array}$} & \multirow{2}{*}{$\begin{array}{c}\text { Erro } \\
\text { Relativo }(\%) \\
\text { E }\end{array}$} \\
\hline & Espectrofotometria & FIA & \\
\hline Descon & $159,7 \pm 0,7$ & $160,8 \pm 0,6$ & $+0,7$ \\
\hline Resfenol & $100,1 \pm 0,5$ & $100,1 \pm 0,9$ & 0,0 \\
\hline Resprin & $201,1 \pm 0,8$ & $201,3 \pm 0,9$ & $+0,1$ \\
\hline Tylenol & $199,7 \pm 0,1$ & $200,1 \pm 0,2$ & $+0,2$ \\
\hline Vick Pyrena* & $500,1 \pm 0,3$ & $499,1 \pm 0,4$ & $-0,3$ \\
\hline
\end{tabular}

'média \pm estimativa do desvio padrão de 6 medidas

*amostra sólida

\section{CONCLUSÕES}

Os estudos realizados neste trabalho evidenciaram a viabilidade do emprego do método espectrofotométrico em fluxo para determinação de paracetamol (acetaminofeno) após sua reação de oxidação com hipoclorito de sódio formando o $\mathrm{N}$-acetil-p-benzoquinonaimina que reage com salicilato em solução básica produzindo um composto indofenólico de coloração azul estável, com forte absorção em $640 \mathrm{~nm}$ no intervalo de $\mathrm{pH}$ entre 9,5 e 10,0

Os resultados obtidos empregando o método proposto estão de acordo com os resultados obtidos usando o método recomendado na literatura e apresentou boa precisão, boa reprodutibilidade e sensibilidade. Com esta metodologia desenvolvida determinou-se paracetamol em diversas amostras comerciais. Além disso, o método apresentou boa freqüência analítica $\left(80 \mathrm{~h}^{-1}\right)$ empregando apenas um volume de $250 \mu \mathrm{L}$ da solução de amostra e ácido salicílico em substituição a solução de fenol, constituindo assim em um procedimento analítico de menor toxicidade.

\section{AGRADECIMENTOS}

Os autores agradecem o apoio financeiro recebido do $\mathrm{CNPq}$, CNPq/PADCT e FAPESP, bem como a bolsa de doutorado do CNPq do aluno C. Aniceto.

\section{REFERÊNCIAS}

1. Korolkovas, A.; Burckhalter, J. H.; Química Farmacêutica, Guanabara Dois: Rio de Janeiro, 1982, p. 159-197.

2. The United States Pharmacopeia - The National Formulary - USP-23; NF18, 1995, p. 16-28. 
3. Official Methods of Analysis of the AOAC - Association of Official Analytical Chemists, Inc.: Arlington, 1984, 40a ed., p. 720-721.

4. Moore, T. J.; Nam, G. G.; Pipes, L. C.; Coury, L. A.; J. Anal. Chem. 1994, 66, 3158 .

5. Girish-Kumar, K.; Letha, R.; J. Pharm. Biomed. Anal. 1997, 15, 1725.

6. Koukli, I. I.; Calokerinos, A. C.; Hadjiioannou, T. P.; Analyst 1989, 114 , 711.

7. Nagaraja, P.; Srinivasa-Murthy, K. C.; Rangappa, K. S.; J. Pharm. Biomed. Anal. 1998, 17, 501.

8. Vilchez, J. L.; Blanc, R.; Avidad, R.; Navalon, A.; J. Pharm. Biomed. Anal. $1995,13,1119$.

9. Cha, C. S.; Chen, J.; Liu, P. F.; Biosens. Bioelectron. 1998, 13, 87.

10. Zen, J. M.; Ting, Y. S.; Anal. Chim. Acta 1997, 342, 175.

11. Walash, M. I.; El Brashy, A. M.; Sultan, M. A.; Mikrochim. Acta 1994, $113,113$.

12. Vyas, P. N.; Kharat, R. B.; Indian J. Pharm. Sci. 1988, 50, 279.

13. Bramwell, H.; Cass, A. E. G.; Gibbs, P. N. B.; Green, M. J.; Analyst 1990, $115,185$.

14. Fang, Y. Z.; Long, D. J.; Ye, J. N.; Anal. Chim. Acta 1997, 342, 13.

15. McCormick, C. P.; Shihabi, Z. K.; J. Liq. Chromatogr. 1990, 13, 1159.

16. Spurway, T.; Wilson, I. D.; Ruane, R. J.; Warrander, A.; J. Planar Chromatogr. - Mod. TLC 1990, 3, 51.

17. Shinde, V. M.; Tendolkar, N. M.; Desai, B. S.; J. Planar Chromatogr. Mod. TLC 1994, 7, 50
18. Markovic, S.; Kusec, Z.; Pharmazie 1990, 45, 935.

19. Patil, S. T.; Sundaresan, M.; Bhoir, I.C.; Bhagwat, A. M.; Talanta 1998, 47,3 .

20. Canada, M. J. A.; Reguera, M. I. P.; Medina, A. R.; Cordova, M. L. F. de; Diaz, A. M.; J. Pharm. Biochem. Anal. 2000, 22, 59.

21. Bouhsain, Z.; Garrigues, M. R. A.; delaGuardia, M.; Anal. Chim. Acta 1996, $330,59$.

22. Verma, K. K.; Jain, A.; Stewart, K. K.; Anal. Chim. Acta 1992, 261, 261.

23. Ramos, M. L.; Tyson, J. F.; Curran, D. J.; Anal. Chim. Acta 1998, 364, 107.

24. Murillo-Pulgarin, J. A.; Garcia-Bermejo, L. F.; Anal. Chim. Acta 1996, 333, 59.

25. Alapont, A. G.; Zamora, L. L.; Martínez-Calatayud, J.; J. Pharm. Biochem. Anal. 1999, 21, 311.

26. Galvez, A. M.; Mateo, J. V. G.; Martínez-Calatayud, J.; Anal. Chim. Acta 1999, 396, 161.

27. Danet, A. F.; David, V.; David, I.; Rev. Roum. Chim. 1998, 43, 811

28. Bouhsain, Z.; Garrigues, M. R. A.; delaGuardia, M.; Analyst 1996, 121, 635.

29. Murfin, J. W.; Analyst, 1972, 97, 663.

30. Farmacopéia Brasileira, 3a ed.. Organização Andrei Ed. S. A.: São Paulo, 1977, p. 656-658. 